\title{
Spatial Estimation of Soil Organic Matter in Karst Peak-cluster Depression
}

\author{
Li Hui ${ }^{1, *}$, Jiang Zhong-Cheng ${ }^{2}$,Yang Qi-Yong ${ }^{3}$, Yin Hui ${ }^{1,2}$ and Wang Yue ${ }^{3}$
}

${ }^{1}$ Institute of Karst Geology, Chinese Academy of Geological Sciences, Guilin, Guangxi 541004, China; ${ }^{2}$ Tourism Department, Huizhou University, Huizhou, Guangdong 516007, China; ${ }^{3}$ College of Environment \& Resources, Guangxi Normal University, Guilin, Guangxi 541004, China

\begin{abstract}
In order to enhance the accuracy of spatial estimation of soil organic matter (SOM), spatial predictions of SOM in 0 20 cm depth were conducted in Guohua Ecological Experimental Area of Minister of Land and Resource of the People's Republic of China. Analysis of multiple linear stepwise regressions showed that the two terrain attributes of relief degree of land surface (RS) and distance from ridge of mountains (DFR) entered into the regression equation. Therefore, RS and DFR were selected as auxiliary variables to predict SOM by MCOK and RK methods. The accuracy of spatial estimation of SOM was compared among methods of ordinary kriging (OK), multivariable cokriging (MCOK) and regression kriging (RK). Results showed that RK and MCOK methods with terrain attributes as auxiliary variables could enhance the accuracy of spatial estimation of SOM, and MCOK method could promote the accuracy notable by $31.33 \%$. This study can provide a new idea and method for evaluation of soil quality in karst areas.
\end{abstract}

Keywords: Auxiliary variable, cokriging, regression kriging, soil organic matter, terrain attribute.

\section{INTRODUCTION}

Soil organic matter (SOM) is the source of plant mineral nutrition and organic nutrition, which is of great significance in the aspects of regional soil fertility, agricultural production and environmental protection [1]. The precise acquisition of the spatial distribution of SOM content information is the basic requirement not only for the accurate mastery of the soil fertility status in karst areas, but also for the scientific management of soil nutrient and regional environmental protection. The most effective method to study spatial variation is geostatistical method [2, 3], which can present the best linear-unbiased-estimator among a limited area of the variable region. Based on auxiliary variables, geostatistical method can effectively enhance the prediction accuracy of soil properties, or reduce the number of sampling points in the case of acceptable accuracy [4-6]. The * staggered distribution of the positive and negative terrains (P-N terrains), combined with the complexity and variability of terrains and physiognomies, brings great difficulties and obstacles to the field survey of the soil status in the peak-cluster-depression karst areas. Therefore the auxiliary-variables-based spatial prediction of soil properties in the peak-cluster-depression karst areas can not only reduce the workload of field soil survey, but also slash expenditures. The practice of enhancing the spatial prediction accuracy through the terrain parameters (as auxiliary-variables) acquired from the calculation by Digital Elevation Model (DEM) receives wider and wider application in spatial predication of soil properties. The main

*Address correspondence to this author at the Institute of Karst Geology, Chinese Academy of Geological Sciences, Guilin, Guangxi 541004, China; Tel: +8613607738053; E-mail: zhjiang@karst.ac.cn reasons for this trend can be divided into three aspects: (1) the terrain parameters acquired from the calculation by DEM, which correlate with soil properties well, are suitable auxiliary variables; (2) the data from DEM is large and continuous in the spatial distribution; (3) the data from DEM is more readily available, and inexpensive.

The current methods to enhance the accuracy of spatial estimation through auxiliary variables mainly include Cokriging (COK) and Regression Kriging (RK). The study by Yate and Warrick [7-10]; Triantafilis [11] et al. showed that when the correlation coefficient is greater than 0.5 between the main and auxiliary variables, COK method can effectively enhance the spatial predictive accuracy of the main variables, but when it's less than 0.5 , the prediction accuracy increase is not obvious. However, the research by Li Wenjun et al. [12] showed that when the correlation coefficient of the main and auxiliary variables is less than 0.5 , the spatial prediction accuracy of the main variables can still be enhanced significantly through the increased auxiliary variables and the application of Multiple Cokriging (MCOK) method. Zhang et al. [13], with terrain parameters as auxiliary variables, effectively enhanced the spatial prediction accuracy of soil organic matter and total nitrogen through the application of RK method. The study by Simbahan et al. [14], Sumfleth and Duttmann [15] showed that the application of RK can effectively enhance the spatial prediction accuracy of soil organic carbon and soil organic matter.

Although both RK and MCOK method can enhance the accuracy of spatial estimation of the main variables, few reports about the contrastive research between the prediction accuracy of RK and MCOK method are found through the literature retrieval. Therefore, this paper takes the typical the 
peak-cluster-depression karst areas (in Guangxi Rocky Desertification-Guohua Ecological Experimental Area of Minister of Land and Resource of the People's Republic of China) as its research area and the terrain parameters as auxiliary variables. Based on the existing researches, this paper compared the accuracy of spatial estimation of SOM among methods of $\mathrm{OK}, \mathrm{MCOK}$ and $\mathrm{RK}$ to determine the best method for the researches of the accuracy of spatial estimation of regional SOM, which can provide a new method for the survey and evaluation of soil quality in the peak-cluster-depression karst areas.

\section{MATERIALS AND METHODS}

\subsection{Study Area}

Guohua Karst Ecological Experimental Area (40¹4'$40^{\circ} 48^{\prime} \mathrm{N}, 116^{\circ} 41^{\prime}-117^{\circ} 30^{\prime} \mathrm{E}$ ), located in Southwest Pingguo County in Guangxi Zhuang Autonomous Region (Fig. 1), was founded in 2001. Topography in this area is characterized with typical peak-cluster-depression karst landscape. The research area, selected from Guohua Karst Ecological Experimental Area, is a rectangular area of 10.8 $\mathrm{km} 2$, the altitude of which is between $150 \sim 530 \mathrm{~m}$, with a mean annual precipitation of $1400 \sim 1550 \mathrm{~mm}$ and winter temperature above $10{ }^{\circ} \mathrm{C}$. The main types of soil in this area are red soil, latosol, yellowish red soil and brown rendzina. The ecological environment in this area was very fragile in the past, which has been improved after the control of rocky desertification. However rocky desertification is still very serious in the neighboring areas.

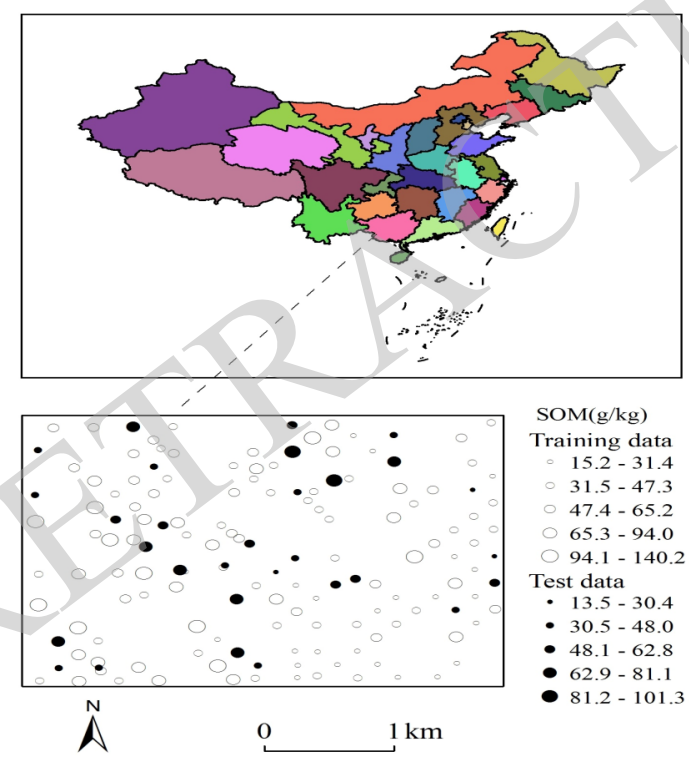

Fig. (1). Location of the research area and the distribution of sample sites in the research area

\subsection{Collection and Mensuration of Soil Sample}

A grid distribution of $140 \mathrm{~m} \quad 140 \mathrm{~m}$ rectangle research area was deployed in ArcGIS. The grid coordinates of the potential sampling sites were put into the global positioning system (GPS) to navigate the field soil sampling. The soil samples of $0-20 \mathrm{~cm}$ were collected by the stainless-steelthrust soil sampler, with the sampling coordinates thoroughly recorded. In the process of sampling, some sampling sites are located on the roads, the buildings or too steep to reach, so the sampling is adjusted to a neighboring area of $10 \mathrm{~m}$ in radii, and the unadjustable sites are discarded. Under the guidance of the principles mentioned above, a total of 149 soil samples were obtained from the research area.

The samples were sent to the laboratory for the removal of contaminations (grass roots and gravels) and natural airdrying. The content of SOM of the samples was determined through the potassium dichromate-wet combustion procedure (NSS, 1995).

\subsection{Data Processing and Analysis}

Supported by GIS technology, a comparative study was made on spatial prediction of SOM in the research area and it proceeded mainly in the following steps.

First, the database of SOM in the research area was founded.

Second, through the spatial analysis by GIS, the sample data was randomly divided into two databases: the modeling database and the validation database.

Third, a multiple stepwise regression analysis about SOM and terrain variables was conducted through the modeling database, in which terrain variables in the regression model were taken as auxiliary variables for Cokriging (COK) and Regression Kriging (RK) analysis.

Fourth, Cokriging (COK) and Regression Kriging (RK) analysis of the spatial prediction of SOM in the research area were respectively carried out through the modeling database.

Fifth, through the validation database, the accuracy of each prediction method was verified for the optimal method of spatial prediction of SOM in the research area.

The software used in this research included ENVI 4.6, ArcGIS9.2, GS+7.0 and SPSS16.0.

\subsubsection{Calculation of Terrain Indices}

A 10 m-resolution DEM was derived from a Digital Topographic Map $(1: 10,000)$ of the research area. And then, the terrain indices were calculated by ArcGIS. Two groups of terrain indices were derived from the DEM: (1) the primary terrain indices, including elevation $H(\mathrm{~m})$, slope $\beta\left(^{\circ}\right)$,aspect angle $S\left(^{\circ}\right) .(2)$ the derivational terrain indices, including catchment area $(A s)$, wetness index $(T W I)$, lengthslope factor $(L S)$, terrain volatility (RS) and distance to ridge of mountains $(D F R)$.

The TWI and LS are defined as:

$$
V_{T W I}=\ln \left(\frac{A_{s}}{\tan \beta}\right)
$$

$$
V_{L S}=\left(\frac{A_{s}}{22.13}\right)^{0.6}\left(\frac{\sin \beta}{0.0896}\right)^{1.3}
$$

In the formula above, $A s$ is the especial catchment area $\left(\mathrm{m}^{2}\right)$ on the contour length, $\beta$ is the value of slope. 


\subsubsection{Regression Kriging (RK)}

The RK approach can be divided into three main aspects: (1) Regression models of the spatial characteristics of SOM are founded, through which the distribution map of regression prediction of SOM in the research area are acquired; (2) The residual between observation data and prediction data from regression model are calculated, by which the spatial distribution map of residual is acquired after RK analysis of the residual. (3) An overlay analysis between the distribution map of regression prediction of SOM and distribution map of residual is conducted for the prediction map of regression kriging in the research area. Prediction results from Regression Kriging analysis can be expressed as:

$$
\mathrm{Z}^{*}=\mathrm{Z}_{p r}^{*}+\varepsilon^{*}
$$

In this formula, $Z^{*}$ is the observation data of SOM; $Z_{p r}^{*}$ is prediction data from multiple regressions; $\varepsilon^{*}$ is the residual between observation data and prediction data.

\subsubsection{Multiple Cokriging}

Multivariate Cokriging analysis is supported by ArcGIS9.2, which permits 4 auxiliary variables at best. To facilitate illustration, the main variables and auxiliary variables are respectively defined as $\mathrm{V} 0$ and $\mathrm{Vi}(\mathrm{i}=1,2 \ldots \mathrm{n})$. In this research, the main variables are SOM, and the auxiliary variables are the terrain indices in the multiple regression model. Multivariate Cokriging analysis by ArcGIS mainly follows four steps [12].

(1) the semi-variance function simulation of the main variables and auxiliary variables are conducted respectively to get the semi-variance function of $\mathrm{V} 0, \mathrm{~V} 1$, $\mathrm{V} 2, \ldots, \mathrm{Vn}$

(2) the covariance function simulation of the main variables and auxiliary variables are conducted respectively to get the semi-variance function of $0-\mathrm{V} 1, \mathrm{~V} 0-\mathrm{V} 2, \ldots, \mathrm{V} 0-$ $\mathrm{Vn}$

(3) the co-semi-variance function analysis is conducted between the covariates, that is, the respective co-semivariance function analysis of $\mathrm{Vi}-\mathrm{Vi}+1, \mathrm{Vi}-$ $\mathrm{Vi}+, \ldots, \mathrm{Vi}-\mathrm{Vn}$, which will produce the relevant covariance function.

(4) cokriging spatial predication is conducted, in which the predication accuracy will be evaluated as well.

\subsubsection{Verification of Prediction Accuracy}

The verification indexes can be mainly divided into two types:

(1) The correlation coefficient, which reflects the similarity degree of the predictive value and the measured value. The closer is the coefficient to 1 , the higher prediction accuracy there will be.

(2) The root mean square error (RMSE), the value of which is between 0 and 1 . The closer is the value to 0 , the higher prediction accuracy there will be.

In order to make a comparison between RK method and MCOK method more visually, this research, with OK method as frame of reference and RMSE as index, respectively calculated the accuracy improvement degree of RK and MCOK in contrast to OK method [15]. The calculation formula is as follows:

$I=\frac{R_{r e f}-R_{x}}{R_{r e f}} \times 100 \%$

In the formula above, $R_{\text {ref }}$ is the root mean square error of reference methods, $R_{x}$ is the root mean square error of other methods. The positive value of I suggested the improvement of accuracy in contrast to the reference methods. The bigger the value of precision, the higher improvement of accuracy will be. On the contrary, if the value of I is negative, the predication accuracy will be lower than the reference methods.

\section{RESULTS AND ANALYSIS}

3.1. The Relationship between SOM and Terrain Variables in the Research Area

In Arcgis, samples were randomly divided into the modeling data set and validation data set, in which the validation data accounts for $20 \%$ of the total samples. The results of statistical analysis for the two data sets of SOM are shown in Table 1. From Table 1, it is indicated that the deviation value for the SOM in the modeling data set is 1.02 , and the kurtosis value is 3.59 , which presents an abnormal distribution. After logarithmic transformation, the deviation value is reduced to -0.02 and the kurtosis value is reduced to 2.47 , which presents a normal distribution and is suitable for statistical semi-variance function analysis. Therefore, the geostatistical analysis below was conducted by the logtransformed data of SOM.

Table 1. Statistical characteristics of soil organic matter.

\begin{tabular}{|c|c|c|c|}
\hline \multirow{2}{*}{ Parameters } & \multicolumn{3}{|c|}{ Data } \\
\cline { 2 - 4 } & $\begin{array}{c}\text { Total } \\
\text { Data }\end{array}$ & $\begin{array}{c}\text { Modeling } \\
\text { Data }\end{array}$ & $\begin{array}{c}\text { Verifying } \\
\text { Data }\end{array}$ \\
\hline \hline Number & 149 & 119 & 30 \\
\hline Mean $/\left(\mathrm{g} \cdot \mathrm{kg}^{-1}\right)$ & 53.2 & 52.9 & 54.2 \\
\hline Minimum $/\left({\left.\mathrm{g} \cdot \mathrm{kg}^{-1}\right)}^{*}\right)$ & 13.5 & 15.2 & 13.5 \\
\hline Maximum $/\left(\mathrm{g}^{\mathrm{k}} \mathrm{kg}^{-1}\right)$ & 140.2 & 140.2 & 101.3 \\
\hline Standard deviation $/\left(\mathrm{g} \cdot \mathrm{kg}^{-1}\right)$ & 25.1 & 26.4 & 19.3 \\
\hline Variation coefficient/ $(\%)$ & 47.2 & 49.9 & 35.6 \\
\hline Deviation Value & 0.95 & 1.02 & 0.23 \\
\hline Kurtosis Value & 3.68 & 3.59 & 2.98 \\
\hline
\end{tabular}

The SOM of the research area had significant correlation with five of the eight chosen terrain indexes (Table 2). SOM had significant negative correlation with both TWI and DFR, with the correlation coefficient as $-0.266(\mathrm{P}<0.01)$ and $-0.475(\mathrm{P}<0.01)$ respectively. While SOM had significant positive correlation with both $\mathrm{H}, \beta$ and $\mathrm{RS}$, with the correlation coefficient as $0.211(\mathrm{P}<0.01), 0.428(\mathrm{P}<0.01)$ and0.494 $(\mathrm{P}<0.01)$ respectively, which presented some similarities to the existing research results $[7-8,13,16]$. 
Table 2. Correlations between SOM and terrain indexes.

\begin{tabular}{|c|c|}
\hline Terrain Indexes & SOM $(\mathbf{g} / \mathbf{k g})$ \\
\hline \hline $\mathrm{H} /(\mathrm{m})$ & $0.211^{*}$ \\
\hline$\beta /\left(^{\circ}\right)$ & $0.428^{* *}$ \\
\hline $\mathrm{S} /\left(^{\circ}\right)$ & 0.042 \\
\hline $\mathrm{AS} /(\mathrm{m} 2)$ & -0.134 \\
\hline $\mathrm{TWI}$ & $-0.266^{* *}$ \\
\hline $\mathrm{LS}$ & -0.031 \\
\hline $\mathrm{RS} /(\mathrm{m})$ & $0.494 * *$ \\
\hline DFR/ (m) & $-0.475^{* *}$ \\
\hline \multirow{2}{|c|}{$*$ Correlation is significant at the 0.05 level (2-tailed). } \\
$* *$ Correlation is significant at the 0.01 level (2-tailed). \\
\hline \multicolumn{2}{|c}{}
\end{tabular}

\subsection{The Determination of Instrumental Variables}

The stepwise regression analysis was conducted, with SOM as dependent variable and the eight topographic indexes as the independent variable. The analysis results showed that the SOM of the research area can be characterized by two multivariate regression model types: (5) and (6)

$\mathrm{VSOM}=32.158+0.56 \mathrm{VRS}$

$\left(\mathrm{R}^{2}=0.244, \mathrm{P}<0.001\right)$

$\mathrm{VSOM}=48.318+0.382 \mathrm{VRS}-0.761 \mathrm{VDFR}$

$\left(\mathrm{R}^{2}=0.307, \mathrm{P}<0.001\right)$

In type (5), only the terrain variable of RS entered the regression model, and the model could explain $24.4 \%$ of the SOM variations.

In type (6), the terrain variables of both RS and DFR entered the regression model, and the model could explain $30.7 \%$ of the SOM variations

Li Wen-jun etc. [12] showed that in MCOK analysis, the prediction accuracy of MCOK was higher than that of $\mathrm{COK}$. While the research of SOM predication by Yang etc. [16] showed that the higher variation degree the multivariate regression model had explained, the higher prediction accuracy of RK method was. Therefore, from the standpoint of prediction accuracy improvement and the reduction of calculation workload, this research selected model (6) and the terrain factors of RS and DFR as auxiliary data of RK and MCOK for the comparative analysis of prediction accuracy of SOM in the research area.

\subsection{Spatial Predictions of SOM Distribution}

\subsubsection{Kriging Prediction of SOM}

Fig. (2a) is the semi-variance function and model parameters of SOM in the research area. The semi-variance function of SOM in the research area is established by the spherical model. C0/Sill is 0.206 and the range is $1990 \mathrm{~m}$, which indicate that SOM in the research area has a strong spatial variability. The coefficient of determination is 0.66 , greater than 0.5 , which indicate that semi-variance function model can well reflect the spatial variability of SOM in the research area [17]. Fig. (3a) is the spatial prediction results of SOM from Ordinary Kriging (OK) method.

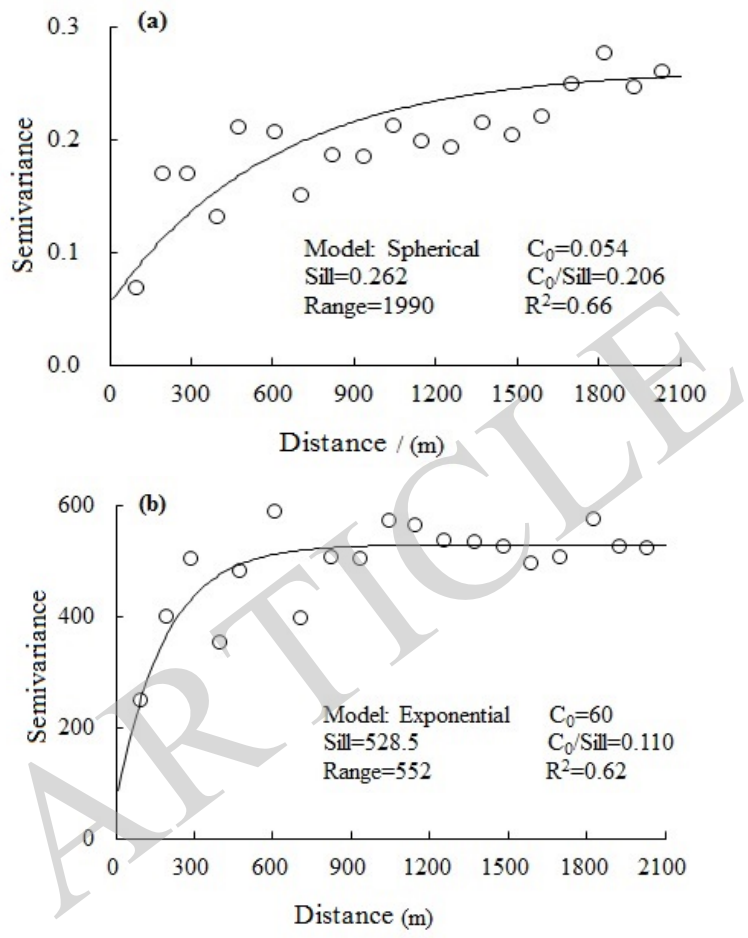

Fig. (2). Theoretical model and parameter of SOM (a) and residual (b).

\subsubsection{Regression Kriging Prediction of SOM}

With regression model 2,inversion operation of SOM in the research area was conducted and residual calculation of the sampling points showed that the residual value ranged from $-35.1 \mathrm{~g} / \mathrm{kg}$ to $74.93 \mathrm{~g} / \mathrm{kg}$, with the average value as $0.01 \mathrm{~g} / \mathrm{kg}$. Residual deviation value was -0.33 , which presented a normal distribution. The variation function of residuals was shown in Fig. (2b). Compared with SOM, residual of $\mathrm{C} 0 / \mathrm{Sill}$ is reduced to 0.110 and the range is reduced to $552 \mathrm{~m}$, which indicated that spatial variation of residual is more intense after considering the terrain variables. In fact, the research area is an area of peak cluster depression with complex and variable physiognomy types, so the size of peak cluster and depression is not large, rarely larger than $1 \mathrm{~km}$ in diameter. Because variable range of SOM overstepped the scale of peak cluster depression, but the variable range of residuals is smaller than the scale of peak cluster depression, therefore, the function of residual variation can reflect the characteristics of the spatial structure in peak cluster depression areas. Fig. (3b) is the RK spatial prediction results of SOM in the research area.

\subsubsection{Multiple Cokriging Prediction of Soil Organic Matter}

With the spherical model selected as the optimal theoretical model, repeated simulations were conducted in GS+ to obtain parameters of semi-variance function and cosemi-variance function between SOM and auxiliary variables. The parameters were summarized in the following 
table (Table 3). As it was shown in Table 3 that the coefficient of determination for semi-variance function model of SOM and terrain indexes ranged from 0.66 to 0.82 , which indicated that the model selected met the requirements. SOM has strong spatial autocorrelation, and the terrain factors respectively have moderate spatial autocorrelations, but the collaborative variation function between SOM and terrain factors has a stronger spatial correlation.

With the spherical model selected in ArcGIS, interpolation of the parameters of each model in Table 3 was conducted to acquire the MCOK spatial predication map of SOM in the research area, which was shown in Fig. (3c).

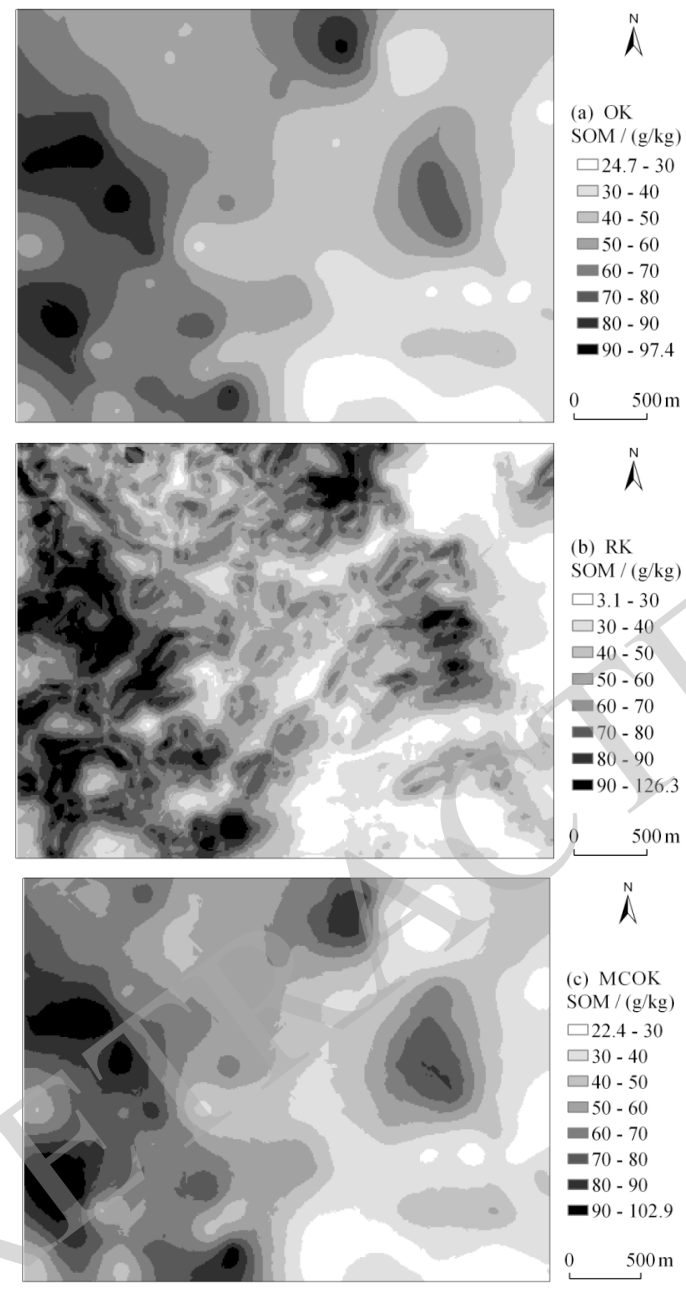

Fig. (3). Distribution Map of SOM by OK (a), RK (b) and MCOK (c) Methods.

It was shown by the spatial distribution of SOM prediction that the general spatial distribution trend of SOM is consistent in the three interpolation figures. The high value of SOM was mainly distributed in the west and southwest, while the low value area was mainly distributed in the east. From the interpolation figures, it was indicated that the amplitude of variation for RK predication was the largest, which ranged from $3.1 \mathrm{~g} / \mathrm{kg}$ to $126.3 \mathrm{~g} / \mathrm{kg}$, while the amplitude of variation for $\mathrm{OK}$ predication was the smallest which ranged from $24.7 \mathrm{~g} / \mathrm{kg}$ to $97.4 \mathrm{~g} / \mathrm{kg}$.
Table 3. Theoretical model and parameter of SOM.

\begin{tabular}{|c|c|c|c|c|}
\hline variable & $\boldsymbol{C}_{\mathbf{0}}$ & Sill & $\boldsymbol{C}_{\mathbf{0}} /$ Sill & $\boldsymbol{R}^{\mathbf{2}}$ \\
\hline \hline$V_{S O M}$ & 0.054 & 0.262 & 0.206 & 0.66 \\
\hline$V_{R S}$ & 110.7 & 328.00 & 0.338 & 0.75 \\
\hline$V_{D F R}$ & 48.9 & 100.8 & 0.485 & 0.82 \\
\hline$V_{S O M^{-}} V_{R S}$ & 0.054 & 4.752 & 0.011 & 0.77 \\
\hline$V_{S O M^{-}} V_{D F R}$ & 0.054 & 2.326 & 0.023 & 0.75 \\
\hline$V_{R S^{-}} V_{D F R}$ & 110.7 & 216.9 & 0.510 & 0.69 \\
\hline
\end{tabular}

\subsubsection{The Comparison Results of Different Methods for Predicting Soil Organic Matter}

The evaluate accuracy of three kinds of interpolation results by using the 30 verifying data of SOM is shown in Table 4.

Table 4. Precision evaluation indexes of different methods for predicting soil organic matter.

\begin{tabular}{|c|c|c|c|}
\hline Parameter & OK & RK & MCOK \\
\hline \hline$r$ & 0.48 & 0.62 & 0.75 \\
\hline RMSE & 24.22 & 20.44 & 16.63 \\
\hline$I(\%)$ & - & 15.61 & 31.33 \\
\hline
\end{tabular}

For the correlation coefficient (r), OK interpolation method is the minimum, MCOK is the maximum; for the root mean square error (RMSE), $\mathrm{OK}$ is the maximum, and $\mathrm{MCOK}$ is the minimum. The results show that the accuracy of the $\mathrm{OK}$ is the lowest, MCOK is the highest, and the RK is in the middle. For I, compared with the OK method, RK method and MCOK method can improve the prediction accuracy of SOM, and MCOK improve the highest rate, up to $31.33 \%$. Therefore, a higher correlation between the main variables and auxiliary variables would be the key to improve the precision of RK method.

\section{CONCLUSION}

(1) The average value of soil organic matter content in the research area is $53.2 \mathrm{~g} / \mathrm{kg}$, and the maximum value is $140.2 \mathrm{~g} / \mathrm{kg}$, while the minimum is only $13.5 \mathrm{~g} / \mathrm{kg}$. SOM has moderate variability. The high value areas of SOM are mainly distributed in the west and southwest of the research area.

(2) Significant correlations were found between SOM and the five terrain indices in the research area. The multiple regression analysis showed that $\mathrm{RK}$ method with terrain fluctuation indexes and the horizontal distance from the mountain divide as auxiliary variables could explain $31.33 \%$ of variation of SOM.

(3) It was shown from the research results that with terrain fluctuation indexes and the horizontal distance from the mountain divide as auxiliary variables, both RK and 
MCOK methods could enhance the accuracy of spatial predication accuracy of SOM, while MCOK method could promote the spatial predication accuracy more notably.

\section{CONFLICT OF INTEREST}

The authors confirm that this article content has no conflict of interest.

\section{ACKNOWLEDGEMENTS}

This work is supported the Ministry of Science and Technology of China (2010BAE00739), the Guangxi Natural Science Foundation (No.2013GXNSFBA019222, 2012GXNSFAA053186).

\section{REFERENCES}

[1] LI Qiquan, Wang Changquan, YUE Tianxiang, LI Bing, Zhang Xin, GAO Xuesong, ZHANG Yi, YUAN Dagang. "Prediction of distribution of soil organic matter based on qualitative and quantitative auxiliary variables: a case study in Santai County in Sichuan Province", Progress in Geography, vol. 33, no.2, pp. 259269, 2014.

[2] XIE Zheng-miao, LI Jing, XU Jian-ming, WU Wei-hong. "Spatial distribution character of fluorine element in soils on Hang-Jia-Hu Plain”, China Environmental Science, vol. 25, no. 6, pp. 719-723, 2005.

[3] J. W. Lv, and X. N. Liu, "Geostatistical analysis on spatial variability of suspended solids concentration in the coast water of Hongkong", China Environmental Science, vol. 34, no. 3, pp. 734$741,2014$.

[4] Ghadermazi Jamshid, Sayyad Gholamabbas, Mohammadi Jahangard "Spatial prediction of nitrate concentration in drinking water using $\mathrm{pH}$ as auxiliary Cokriging variable", Procedia Environmental Sciences, vol. 3, pp. 130-135, 2013.

[5] O. Babak, and C. V. Deutsch, "Improved spatial modeling by merging multiple secondary data for intrinsic collocated cokriging", Journal of Petroleum Science and Engineering, vol. 69, pp. 93-99, 2009 .

[6] Wu C, Wu J, Luo Y, Zhang L, DeGloria SD. "Spatial prediction of soil organic matter content using cokriging with remotely sensed data", Soil Science Society of America Journal, vol. 73, no. 4, pp. 1202-1208, 2009.

[7] Pei, T., C.Z. Qin, A.X. Zhu, L. Yang, M. Luo, B.L. Li, C.H. Zhou, "Mapping soil organic matter using the topographic wetness index: a comparative study based on different flow-direction algorithms and kriging methods", Ecological Indicator, vol. 10, no. 3, 610619, 2010.

[8] Zhang Shiwen, Huang Yuanfang, Shen Chongyang, "Spatial prediction of soil organic matter using terrain indices and categorical variables as auxiliary information", Geoderma, vol.171, pp. 35-43, 2012.

[9] Q. Y. Yang, F. W. Zhang, Z. C. Jiang, "Relationship between soil depth and terrain attributes in karst region in Southwest China", Journal of Soils and Sediments, pp. 1568-1576, 2014.

[10] S. R. Yates, and A. W. Warrick, "Estimating soil water content using cokriging", Soil Science Society of America Journal, vol. 51, pp. 23-30, 1987.

[11] J. Triantafilis, and I. Odeh, and A. B. McBratney, "Five geostatistical models to predict soil salinity from electromagnetic induction data across irrigated cotton ", Soil Science Society of America Journal, vol. 65, pp. 869-878, 2001.

[12] W. J. Li_Q. Y. Yang_B. F. Peng, "Spatial Variability of Total Soil Nitrogen Content in Karst Area of Southwest China", Transactions of the Chinese Society for Agricultural Machinery, vol. 45, no.9, pp. 150-154, 2014.

[13] Zhang Sumei, Wang Zongming, Zhang Bai, Song Kaishan, Liu Dianwei, Li Fang, Ren Chunying, Huang Jian, Zhang Huilin, "Prediction of spatial distribution of soil nutrients using terrain attributes and remote sensing data", Transactions of the CSAE, vol 26, no.5, pp. 188-194, 2010.

[14] G. C. Simbahan, A. Dobermann, P. Goovaerts, "Fine-resolution mapping of soil organic carbon based on multivariate secondary data", Geoderma, vol.132, no. 3/4, pp. 471-489, 2006.

[15] K. Sumfleth, and R. Duttmann, "Prediction of soil property distribution in paddy soil landscapes using terrain data and satellite information as indicators", Ecological Indicators, vol. 8, no. 5, pp. 485-501, 2008.

[16] Q. Y. Yang, Z. C. Jiang, W. J. Li, H. Li. "Prediction of soil organic matter in peak-cluster depression region using terrain indices," Soil \& Tillage Research, vol. 114, pp. 126-132, 2014.

[17] M. Duffera, J. G. White, and R. Weisz, "Spatial variability of Southeastern US Coastal Plain soil physical properties: Implications for site-specific management", Geoderma, vol. 137, no. 3, pp. 327-339, 2007.

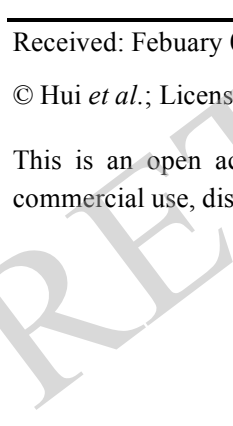

Revised: April 03, 2015

Accepted: May 25, 2015

This is an open access article licensed under the terms of the (https://creativecommons.org/licenses/by/4.0/legalcode), which permits unrestricted, noncommercial use, distribution and reproduction in any medium, provided the work is properly cited. 